\title{
High Temperature Dynamic Hohlraums on the Pulsed Power Driver Z
}

T.J. Nash, M.S. Derzon, G. A Chandler, R. Leeper, D. Fehl, J.

Lash, C. Ruiz, G. Cooper, J.F. Seaman, J. McGurn, S. Lazier, J.

Torres, D. Jobe, T. Gilliland, M. Hurst, R. Mock, P. Ryan, D.

- Nielsen, J. Armijo, J. McKenney, R. Hawn, and D. Hebron

Sandia National Laboratory

Albuquerque, NM, 87123

RECEIVED

J.J. MacFarlane

JAN 211999

U. Wiscensin

OST

Madison, WI, 53705

D. Petersen, R. Bowers, and W. Matuska

Les Alames National Laboratery

Los Alamos, NM, 87545

D.D. Ryutov

Lawrence Livemere Laboratecy

Livermore, CA, 94550

PACS numbers:52.35.Py, 52.55.Ez, 52.70.Kz, 52.70.La 


\section{DISCLAIMER}

This report was prepared as an account of work sponsored by an agency of the United States Government. Neither the United States Government nor any agency thereof, nor any of their employees, make any warranty, express or implied, or assumes any legal liability or responsibility for the accuracy, completeness, or usefulness of any information, apparatus, product, or process disclosed, or represents that its use would not infringe privately owned rights. Reference herein to any specific commercial product, process, or service by trade name, trademark, manufacturer, or otherwise does not necessarily constitute or imply its endorsement, recommendation, or favoring by the United States Government or any agency thereof. The views and opinions of authors expressed herein do not necessarily state or reflect those of the United States Government or any agency thereof. 


\section{DISCLAIMER}

Portions of this document may be illegible in electronic image products. Images are produced from the best available original document. 


\begin{abstract}
In the concept of the dynamic hohlraum an imploding z-pinch is optically thick to its own radiation. Radiation may be trapped inside the pinch to give a radiation temperature inside the pinch greater than that outside the pinch. The radiation is typically produced by colliding an outer $Z$-pinch liner onto an inner liner. The collision generates a strongly radiating shock, and the radiation is trapped by the outer liner. As the implosion continues after the collision the radiation temperature may continue to increase due to ongoing PdV (pressure times change in volume) work done by the implosion. In principal the radiation temperature may increase to the point at which the outer liner burns through, becomes optically thin, and no longer traps the radiation. One application of the dynamic hohlraum is to drive an ICF (inertial confinement fusion) pellet with the trapped radiation field. Members of the dynamic hohlraum team at Sandia National Labs have used the pulsed power driver $\mathrm{Z}(20 \mathrm{MA}, 100 \mathrm{~ns})$ to create a dynamic hohlraum with temperature linearly ramping from 100 to $180 \mathrm{eV}$ over $5 \mathrm{~ns}$. On this shot zp214 a nested tungsten wire array of 4 and $2 \mathrm{~cm}$ diameters with masses of 2 and $1 \mathrm{mg}$ imploded onto a $2.5 \mathrm{mg}$ plastic annulus at 5 $\mathrm{mm}$ diameter. The current return can on this shot was slotted. It is likely the radiation temperature may be increased to over $200 \mathrm{cV}$ by stabilizing the pinch with a solid curtent return can. A current return can with 9 slots imprints 9 filaments onto the imploding pinch. This degrades the optical trapping and the quality of the liner collision. A $1.6 \mathrm{~mm}$ diameter capsule situated inside this dynamic hohlraum of $2 \mathrm{p} 214$ would see $15 \mathrm{~kJ}$ of radiation impinging on its surface before the pinch itself collapses to a $1.6 \mathrm{~mm}$ diameter. Dynamic hohlraum shots including pellets are scheduled to take place on $Z$ in September of 1998.
\end{abstract}




\section{Introduction}

The dynamic hohlraum historically has been known as the flying radiation case (1), the imploding liner hohlraum (2), and double liners (3). The main idea of the dynamic hohlraum is to produce radiation by the collision of an outer $z$ pinch liner with an inner liner target, and then trap this radiation during the continued implosion of the system. This trapping of radiation is possible because the combined mass of the two liners may be optically thick. As the inner liner is compressed energy density and radiation temperature increase. There are many applications of dynamic hohlraums to the study of high energy density plasmas for ICF (inertial confinement fusion). $[4,5,6]$ The focus of our -.. dynamic hohlraum research is to drive an ICF capsule. [7]

Dynamic hohlraum temperatures scale favorably with the machine current of the Z-pinch driver. Radiated power and mass driven by a $Z$ pinch both scale as the square of the current. The increased mass with the current improves the opacity and the integrity of the dynamic hohlraum case. Once the mass and current are sufficient to trap the radiation produced by the liner collision, the radiation temperature should scale as the square root of the machine current. To establish the scaling of dynamic hohlraum radiation temperature with current, experiments have been carried out on three Z-pinch drivers: ANGARA ( 3.5 MA), SATURN (7 MA), and Z (20 MA). [8] In this paper we present the 
measurements of dynamic hohlraum radiation temperature on $Z$ and discuss the scaling issues.

$\mathrm{Z}$ is a $40 \mathrm{TW}$ electrical pulsed power machine. $[9,10]$. It drives a $z$-pinch with a $20 \mathrm{MA}$ current with a $100 \mathrm{~ns}$ rise time. The longer rise time with respect to the faster driver SATURN, $50 \mathrm{~ns}$, could cause increased growth of instabilities of the imploding current sheath, and increased $x$-ray pulse widths. Increases in the thickness or instability of the current sheath are detrimental to the performance of a Z-pinch dynamic hohlraum system.

Pinch stability is very important to the performance of a dynamic hohlraum. A stable thin, approximately $1 \mathrm{~mm}$, current sheath is beneficial because it will $\cdots$ produce a sharp collision between the two liners, efficiently creating a radiation field which is heated as the implosion continues. An unstable implosion has a larger effective current sheath up to $5 \mathrm{~mm}$ in width, producing a lower power collision broad in time. A stable pinch also allows compression to less than 1 $\mathrm{mm}$ diameter, which greatly increases the dynamic hohlraum radiation temperature. There is speculation in the literature that using nested wire arrays can reduce the growth of the Rayleigh-Taylor instability and improve the radiated power of the $Z$ pinch. $[11,12]$

Advances in the stabilization of $\mathrm{Z}$ pinches through the use of large-wirenumber arrays have made possible dynamic hohlraum radiation temperatures 
well in excess of $100 \mathrm{eV}$ that we present in this article. $[13,14]$ These advances have included the demonstration of $80 \mathrm{TW}$ of radiation from the accelerator SATURN using a 120-tungsten-wire array, and over $200 \mathrm{TW}$ from $\mathrm{Z}$ using a 240-tungsten wire array. $[15,16]$

Another stabilization technique we introduce in this paper is in the use of a solid can for the current return path. Typically on $Z$ nine slots are cut in the current return can for side-on diagnostic access. These nine slots imprint nine filaments onto the imploding pinch. The use of a solid current return can eliminate these filaments and increase the power radiated out the axis of the pinch.

\section{Experimental Design}

In these experiments on $Z$ a nested wire array with 240 and $1207.5 \mu \mathrm{m}$ diameter tungsten wires at 4 and $2 \mathrm{~cm}$ diameter respectively were imploded onto $5 \mathrm{~mm}$ diameter targets. The array was $1 \mathrm{~cm}$ long and its mass was $3 \mathrm{mg}, 2 \mathrm{mg}$ in the outer tungsten array and $1 \mathrm{mg}$ in the inner nested array. Such a nested array implodes to strike a $5 \mathrm{~mm}$ diameter inner target with a strike velocity in excess of $50 \mathrm{~cm} / \mu \mathrm{s}$. The current return can was $50 \mathrm{~mm}$ in diameter.

For zp214 the current return can was slotted with 9 apertures $8 \mathrm{~mm}$ in width and $8 \mathrm{~mm}$ long. The 9 apertures are cut for side-on viewing of the pinch. We will present data showing that the 9-fold symmetry of the return can imprints 
onto the pinch which develops 9 filaments. The $5 \mathrm{~mm}$ diameter target for $\mathrm{zp} 214$ was a plastic annulus weighing $2.5 \mathrm{mg}$.

We shall also present results for $\mathrm{zp} 297$ in which the current return can had no slots. The $5 \mathrm{~mm}$ diameter target for $\mathrm{zp} 297$ was a cylindrical foam at $14 \mathrm{mg} / \mathrm{cc}$ density weighing $3 \mathrm{mg}$.

The load current is measured by B-dot monitors placed in the anode plate tens of $\mathrm{cm}$ from the pinch axis. [17] The implosion time is measured by the time difference between the current rise and rise of the $\mathrm{x}$-ray pulse as measured by a five channel $\mathrm{x}$-ray diode array. The typical load current is $20 \mathrm{MA}$ and the loads for zp214 and zp297 are designed to implode in $100 \mathrm{~ns}$.

The $\mathrm{x}$-ray radiation is measured with carbon photocathode $\mathrm{x}$-ray diodes (XRDs). [18] Five responses are provided by five different filters: $4 \mu \mathrm{m}$ of kimfol, $1 \mu \mathrm{m}$ of vanadium, $.75 \mu \mathrm{m}$ of zinc with 0.5 micron of parylene, $8 \mu \mathrm{m}$ of beryllium with $1 \mu \mathrm{m}$ of parylene, and $9 \mu \mathrm{m}$ of beryllium with $1 \mu \mathrm{m}$ of vanadium. The nominal bandpasses of the above filters are at $250,450,650,850$, and 1400 $\mathrm{eV}$. Two sets of the five-channel $\mathrm{x}$-ray diode arrays were fielded. One views the pinch from the side to record temperatures outside of the outer liner, and the other views the pinch from the axis to measure temperatures inside the inner liner. The XRDs were absolutely calibrated at the National Synchrotron facility at Brookhaven National Laboratory. 
Bolometers are fielded along the axis and from the side of the pinch to measure total radiated yield in these two directions. The response of the bolometers is 1 to $2 \mathrm{~ns}$, and the signal is typically too noisy to be differentiated to give radiated power, but the yield can be used to normalize the XRD signals to the radiated power.

Two microchannel plate framing cameras were fielded on axis to unfold the radial dependence of the radiation temperature as a function of time as the radiation case or $z$-pinch implodes. Each camera recorded 11 time frames and 1 time integrated frame. Each frame recorded two images, one filtered for a $250 \mathrm{eV}$ bandpass, the other for radiation above $800 \mathrm{eV}$. One camera had a magnification of $2 / 3$, the other $1 / 3$. Each frame was gated with a 1 ns pulse. The resolution of these cameras at the pinch is between 200 and $300 \mu \mathrm{m}$. With normalization provided by the on-axis $\mathrm{XRDs}$ and bolometers these cameras make possible the measurement of the spatial dependence of the radiation temperature as a function of time.

We also fielded radially resolved temporally integrated crystal spectrometers side-on and on-axis. These spectrometers measure electron temperature from the slope of the tungsten continuum radiation above energies of $900 \mathrm{eV}$.

Experimental Results 
In Figure 1 we show the load configuration for zp214. As mentioned in the introduction the load is a nested tungsten wire array imploding onto a plastic annulus. For this shot zp214 the current-return can was slotted, and improvement in performance is to be expected if the same load is shot with a solid current-return can. The tungsten wires are $7.5 \mu \mathrm{m}$ in diameter with 240 wires in the outer array at $4 \mathrm{~cm}$ diameter and 120 wires in the inner array at $2 \mathrm{~cm}$ diameter. The masses of the two arrays are $2 \mathrm{mg}$ and $1 \mathrm{mg}$. The collision target is a plastic annulus weighing $2.5 \mathrm{mg}$. As shown in figure 1 the back wall of the dynamic hohlraum is covered with a gold half-moon to provide albedo contrast in the on-axis $\mathrm{x}$-ray images. The axial viewing aperture is $4 \mathrm{~mm}$ in diameter.

The radial dependence of the radiation temperature parameteric in time at $1 \mathrm{~ns}$ intervals is shown in Figure 2. These temperatures are based upon the power radiated out the axis and the axial time-resolved $x$-ray images. From the bolometer and the XRDs the radiated power out the axis on zp297 reaches 12 TW before the implosion stagnates on axis. The measured axial power is shown in Figure 6. Using the axial power, the time-resolved $x$-ray inages may be converted to images in radiation temperature. Line-outs of these temperature images perpendicular to the gold half-moon are shown in Figure 2. As the collison commences at $527 \mathrm{~ns}$ as indicated in the Figure 2, one sees the albedo 
contrast between the gold half-moon at $85 \mathrm{eV}$ and the plastic half-moon at 70 $\mathrm{eV}$.

Because the camera views the hohlraum at an angle of 6 degrees with respect to the axis, it is able to view the wall of the plastic annulus. The line-out at 528 ns in Figure 2 is taken across the wall of the annulus. At 529 ns the plastic wall has reached $100 \mathrm{eV}$, burned through, and can no longer be seen in absorption against the tungsten pinch. At 530 ns some tungsten has blown over the end of the plastic and arrived on axis. This feature has been denoted as the tungsten curtain. The axial diagnostics must look through this tungsten to see into the hohlraum. Several techniques are now being used to minimize this curtain. They -. include 5 degree glide planes to accelerate the tungsten away from the viewing hole, smaller viewing apertures as low as $2 \mathrm{~mm}$, and efforts to minimize any gap between the plastic annulus and the anode plane. As the implosion compresses the hohlraum down to less than $2 \mathrm{~mm}$ diameter at $532 \mathrm{~ns}$ the inside of the tungsten wall has reached $180 \mathrm{eV}$. At this time the hohlraum is still open with a $1.6 \mathrm{~mm}$ diameter. We may project that the temperature inside the hohlraum reached a value greater than $190 \mathrm{eV}$ before the pinch completely collapsed the hohlraum. The last time gate of the time-resolved camera was at 532 ns so we have no measurement of the temperature profile beyond this time on zp214. 
In Figure 3 we present the measurement of the dynamic hohlraum temperature and diameter plotted versus time. After the collision the hohlraum collapses at a velocity of $35 \mathrm{~cm} / \mu \mathrm{s}$. During most of the compression the temperature rises linearly at $20 \mathrm{eV} / \mathrm{ns}$, then rolls over to a rate of $10 \mathrm{eV} / \mathrm{ns}$ at the end of the compression. It is likely that the rate of temperature increase rolls over because the tungsten pinch is becoming optically thin and allowing radiation to escape out the sides of the dynamic hohlraum. We are presently designing fusion pellets at $1.6 \mathrm{~mm}$ diameter. From Figure 3 we see that such a pellet would be separate from the imploding pinch until 532 ns when the temperature has reached $180 \mathrm{eV}$. -.

By integrating the hohlraum flux over the surface area of a $1.6 \mathrm{~mm}$ diameter pellet one arrives at the radiation energy impingent on the capsule as shown in Figure 4. The energy delivered to the capsule surface before the pinch reaches $1.6 \mathrm{~mm}$ in diameter is $15 \mathrm{~kJ}$. This is a considerable amount of energy for driving a pellet and gives reason for optimism in using a $Z$ pinch to drive a fusion pellet.

Because zp214 used a slotted can we also have measurement of the power radiated out the side of the pinch. Using the diameter of the pinch as measured by the on-axis time-resolved camera, we can then unfold radiation temperature both inside and outside of the dynamic hohlraum. These results are presented in Figure 5. The hohlraum is hotter on the inside than the outside for about 3 ns. 
The outside temperature rises abruptly at $531 \mathrm{~ns}$ when the inside temperature is $170 \mathrm{eV}$. At this temperature it is likely that the tungsten wall is becoming optically thin and leaking the hohlraum radiation out the side. The value of the outside temperature at 533 ns, being greater than the inside temperature, is a artifact of the side power measurement which includes other power sources, such as the z-pinch halo.

As we have mentioned $\mathrm{zp} 214$ formed nine filaments due to a slotted current return can. This is not optimal for trapping radiation and forming a good radiation case. With a more cylindrical case one could expect the hohlraum to maintain its integrity to temperatures higher than $170 \mathrm{eV}$. This is one of the benefits of added stability provided by a solid current return can.

The measured power radiated out the axis on $z p 214$ is compared to a simulation in Figure 6. [19] The simulation is a two dimensional $(r, z)$ code. It does not include the possibility of azimuthal filamentation. For this reason the simulation shows a much sharper radiation burst during the collision at 2526 ns than is measured. It also shows a much sharper burst when the pinch reaches axis at $2533 \mathrm{~ns}$. Indeed with the use of a solid current-return can one might expect the power radiated out the axis to improve towards the impressive bursts shown in the simulation. [20] 
Azimuthal filamentation as measured by an axial time-resolved pinhole camera is shown in Figure 7. This data was recorded for $\mathrm{zp} 113$ which was for a single tungsten wire array imploding onto a solid cylindrical foam. Filaments are seen approximately every 40 degrees. The azimuthal line-out was taken at a diameter of $4 \mathrm{~mm}$ as the pinch reached this diameter. One would expect a better radiation case if these filaments could be eliminated with a solid current-return can.

In Figure 8 we present the improvement to be gained by using a solid current return can. Figure 8 compares axial radiated power for two shots, zp210 and zp211, whose only difference was a slotted current-return can and a solid current-return can. The load on these two shots was a $0.75 \mathrm{~cm}$ long nested tungsten wire array at 35 and $17.5 \mathrm{~mm}$ diameter. The target was $2 \mu \mathrm{m}$ thick annular gold at $5 \mathrm{~mm}$ diameter. The mass loading for these shots was relatively heavy with $3.6 \mathrm{mg} / \mathrm{cm}$ of tungsten impacting $5.4 \mathrm{mg} / \mathrm{cm}$ of gold. One sees from figure 8 that the radiated power at collision is improved a factor of 1.7 using the solid current return can. The radiation pulse width at collision with the solid can is a narrow 3.5 ns. We note that the radiation temperature for the dynamic hohlraum of zp211 was flat at $150 \mathrm{eV}$ during the $5 \mathrm{~ns}$ compression of the hohlraum. 
We shall next present results from zp297 which used a solid current return can. The load configuration for $\mathrm{zp} 297$ is shown in Figure 9. The nested tungsten wire array is identical to $\mathrm{zp} 214$. The main difference from $\mathrm{zp} 214$ is in the use of a solid return can. The target is also different, being a cylindrical foam for zp297. The foam density is $14 \mathrm{mg} / \mathrm{cc}$ and weighs $3 \mathrm{mg}$. The viewing aperture for this shot was $2 \mathrm{~mm}$ diameter in an effort to minimize the amount of tungsten blowing past the end of the target to form a tungsten curtain across the line of sight of the axial diagnostics.

The radial temperature profiles for $\mathrm{zp} 297$ parametric in time are shown in Figure 10. The pinch reaches the axis at $541.5 \mathrm{~ns}$ at which time a spot with a radiation temperature of $300 \mathrm{eV}$ is formed. This pinch was not centered in the viewing aperture. It was off by $400 \mu \mathrm{m}$ as indicated in figure 10 at $541.5 \mathrm{~ns}$. This is thought to be due to accuracy in target alignment. We initially record signal out the $2 \mathrm{~mm}$ aperture at a temperature of $100 \mathrm{eV}$ at $537.5 \mathrm{~ns}$. At this time the pinch is still outside the $2 \mathrm{~mm}$ diameter viewing aperture, and we are looking only at foam. At 538.5 ns the pinch has just entered the viewing aperture on one side, the left side of Figure 10, and the foam temperature has risen to $130 \mathrm{eV}$. At $539.5 \mathrm{~ns}$ the tungsten is at $190 \mathrm{eV}$ while the foam is at 170 $\mathrm{eV}$. The last open frame is at $540.5 \mathrm{~ns}$ when the pinch is approximately $800 \mu \mathrm{m}$ in diameter. The temperature at this time is an impressive $230 \mathrm{eV}$. 
Zp297 was much hotter than any other dynamic hohlraum shot on Z, radiating 12 TW out of a $2 \mathrm{~mm}$ diameter hole. By comparison zp214 radiated 12 TW out of a $4 \mathrm{~mm}$ diameter hole. In Figure 11 we compare the on-axis time integrated crystal spectra for $z \mathrm{p} 214$ and $\mathrm{zp} 297$. The spectum is basically tungsten continuum with $\mathrm{M}$ shell absorption features. The slope of the continuum is indicative of electron temperature. On zp214 the electron temperature was $700 \mathrm{eV}$, and there were several $\mathrm{M}$ shell absorption features in the spectrum. On zp297 the electron temperature is a much higher $2 \mathrm{keV}$ and all but one of the absorption features has disappeared. This is likely due to the tungsten burning through most of the $M$ shell on zp297. The large electron temperature on zp297 is likely due to an equilibration of electrons and ions brought about by the high level of stability of this pinch. With higher stability of implosion, higher electron densities are reached at peak compression, and the ions, which carry all the implosion kinetic energy, may equilibrate with the electrons. The radiation temperature is still well below the particle temperature on $\mathrm{Z}$.

The time histories for the pinch diameter and radiation temperature are compared for zp214 and zp297 in Figure 12. For each load the velocity of compression is the same, but the radiation temperature history is very different. The temperature for the plastic annular target of zp214 is up earlier than the 
foam target of $\mathrm{zp} 297$, and rises more slowly. Indeed the temperature profile of zp214 would actually deliver more energy to a $1.6 \mathrm{~mm}$ diameter capsule than that of $z$ 2297. For this reason we intend to use annular targets to drive exploding pusher targets. On the other hand the radiation temperature profile of zp297 is very close to the drive pulse needed for an ablatively driven ICF pellet. For this reason we intend to use foam as the target for pulse shaping experiments.

\section{Scaling}

The scaling of radiation temperature for a dynamic pinch hohlraum goes roughly as the square root of the machine current. This is to be expected since the electrical power which creates the radiated power scales as the current squared. Another factor to consider in the scaling is the integrity of the imploding hohlraum case, whether or not the pinch remains optically thick as the temperature of the hohlraum increases. The pinch mass driven scales as the square of the current. On larger machines the added mass will increase the opacity of the dynamic hohlraum, and permit higher radiation temperatures before the case burns through.

Scaling of the radiation temperature with machine current is shown in Figure 13. The data points are from ANGARA at 3.5 MA, SATURN at $7 \mathrm{MA}$, and $Z$ at $20 \mathrm{MA}$. For each machine the radiation temperature is taken in a dynamic 
hohlraum configuration with a diameter of approximately $1 \mathrm{~mm}$. The machines all had different implosion times, and different levels of integrity in the radiation case. In general the faster pinches are more stable, and the larger machines provide a better radiation case. Despite these differences, the fit through the data points is very close to the square root of the current. One could then expect a $60 \mathrm{MA}$ driver to provide a dynamic hohlraum radiation temperature well over $300 \mathrm{eV}$.

It is likely that a $60 \mathrm{MA}$ driver would implode over a longer time-scale, say $150 \mathrm{~ns}$, because of the limitations in voltage of the insulator stack at the watervacuum interface. On $\mathrm{Z}$ the vacuum inductance is $9 \mathrm{nH}$ with rate of current rise $\cdots$ of $3.5 \times 10^{14} \mathrm{~A} / \mathrm{s}$. This sets the voltage at the insulator stack as $3 \mathrm{MV}$, a value that is also measured routinely on every shot. Indeed insulator breakdown shots have shown the stack to breakdown at 4.6 MV on the water side of the stack. For the same current rise time as $Z$, a $60 \mathrm{MA}$ driver would need to hold off 9 MV at the insulator stack. So the stack on $Z$ is not capable of providing $60 \mathrm{MA}$ with a $100 \mathrm{~ns}$ rise time. Presently the design for a $60 \mathrm{MA}$ driver is to increase the diameter of the stack by roughly $50 \%$ with respect to $Z$, and to increase the implosion time by $50 \%$ as well. These two changes will increase the breakdown voltage to about $10 \mathrm{MV}$, which is enough to drive $60 \mathrm{MA}$. Because a $60 \mathrm{MA}$ driver will implode a wire array more slowly than $Z$, the initial diameter of the 
array will be between 5 and $6 \mathrm{~cm}$. Such an array will experience more growth of the RT instability during implosion. Stabilization techniques such as a triplenested wire array, or foam annuli for snowplow stabilization, will need to be investigated as dynamic hohlraum drivers on a 60 MA machine.

\section{Conclusion}

Using the pulsed power driver $\mathrm{Z}$ (20 MA, $100 \mathrm{ns)}$ we have reached a dynamic hohlraum temperature of $230 \mathrm{eV}$ at a diameter of $800 \mu \mathrm{m}$ for zp297. This is the first recording of a z-pinch driven open hohlraum at a temperature over $200 \mathrm{eV}$. On zp214 we reached a radiation temperature of $180 \mathrm{eV}$ at a diameter of $1.6 \mathrm{~mm}$ and this source would deliver $15 \mathrm{~kJ}$ of radiation to a $1.6 \mathrm{~mm}$ diameter capsule. Although zp297 did have a different central target than zp214, a cylindrical foam as compared to a plastic annulus, the main improvement in the temperature drive was in the use of a solid current-return can. A current-return can with nine slots for side-on diagnostic access imprints nine azimuthal filaments onto the imploding pinch. We have shown that in one pinch configuration replacing the slotted can with a solid can increases the power radiated out the axis a factor of 1.7 . The rapid rise of the radiation temperature on zp297 makes using a cylindrical foam target suitable for driving an ablation capsule. The longer dwell time of radiation on a capsule afforded by an annular target such as on zp214 makes it more suitable for driving exploding pusher 
targets. We anticipate that shooting the load of zp214 with a solid currentreturn can will increase the useable temperature for driving a $1.6 \mathrm{~mm}$ diameter exploding pusher pellet to over $200 \mathrm{eV}$. Since the radiation temperature scales as the square root of the machine current, a $60 \mathrm{MA}$ driver would be capable of achieving radiation temperatures in excess of $340 \mathrm{eV}$.

*Sandia is a multiprogram laboratory operated by Sandia Corporation, a Lockheed Martin Company, for the United States Department of Energy under Contract DE-AC04-94AL85000. 


\section{References}

1. J. Brownell, R.L. Bowers, K.D. McLenithan, and D.L. Peterson, Phys. Plas., 5, No 5, p. 207, 1998

2. M.K. Matzen, T.W. Hussey, G.R. Montry, and J.E. Morel, "A ID Scaling Study of Radiation Sources Using Imploding Liners," submitted for deposit to the National Technical Information Service, Springfield, VA 22161

3. V. P. Smirnov, Plasma Physics and Controlled Fusion, 33, \#13, 1697, (1991)

4. D.D. Ryutov, M.S. Derzon, and M.K. Matzen, "The Physics of Fast Z-Pinches", SAND981632, July, 1998, Available through NTIS, U.S. DOC, 5285 Port Royal Rd., Springfield, VA, 22161

5. M.S. Derzon, T.J. Nash, G.O. Allshouse, A.J. Antolak, C. Deeney, M. Hurst, J.S. McGurn, D.J. Muron, J.F. Seaman, J. MacFarlane, T. Demiris, L. Hrubesh, H. Lewis, D. Ryutov, T. Barber, T. Gilliland, D. Jobe, and S. Lazier, Rev. Sci. Ins., 68, No. 1, p. 848, 1997

6. M.K. Matzen, Phys. Plas. 4, No. 5, 1519. 1997

7. J. Lendl, Phys. Plasmas, 2 (11), November 1995

8. T.J. Nash, M.S. Derzon, G. Allshouse, C. Deeney, J.F. Seaman, J. McGurn, D. Jobe, T. Gilliland, J.J. MacFarlane, P. Wang, and D.L. Petersen, "Dynamic Hohlraum Experiments on SATURN," Proceedings of the 4 th International Conference on High-Density Z-pinches, Vancouver, Canada, May, 1997, American Institute of Physics, Woodbury, New York, 1997, p. 175

9. R. B. Spielman, M.K. Matzen, M.A. Palmer, P.B. Rand, T.W. Hussey, and D.H. McDaniel, Appl. Phys. Lett., 47, 229, (1985).

10. R. B. Spielman, C. Deeney, G. A. Chandler, M. R. Douglas, D. L. Fehl, M. K. Matzen, D. H. McDaniel, T. J. Nash, J. L. Porter, T. W. L. Sanford, J. F. Seamen, W. A. Stygar, K. W. Struve, S. P. Breeze. J. S. McGurn, J. A. Torres, D. M. Zagar, T. L. Gilliland, 
D. O. Jobe, J. L. McKenney, R. C. Mock, M. Vargas. T. Wagoner, and D. L. Peterson. Proc. of the 11 th Int. Conf. on Particle Beams, edited by $K$. Jungwirth and J. Ullschmied, Academy of Sciences of the Czech Republic, Prague, 1996, p. 150

11. D.L. Petersen, R.L. Bowers, J.H. Brownell, A. E. Greene, K.D. McLenithan, T.A. Oliphant, N.F. Roderick, and A.J. Scannapieco, Phys. Plasmas 3 (1), p. 368, January 1996

12. Alexander Velikovich, F.L. Cochran, and J. Davis, Phys. Rev. Lett., 77 (5), p. 853, July 29, 1996

13. M.S. Derzon, et al, "Experimental Resulis and Modeling of a Dynamic Hohlraum on SATURN," SAND98-1190, Available through NTIS, U.S. DOC, 5285 Por Royal Rd., Springfield, VA, 22161

14. T.W.L. Sanford, R.B. Spielman, G.O. Allshouse, G.A. Chandler, D.L. Fehl, W.A. stygar, K.W. Struve, C. Deeney, T.J. Nash, J.F. Seamen, R.C. Mock, T.L. Gilliland, J.S. McGum, and D.O. Jobe, IEEE Transactions on Plasma Science, 26, 44, P 1086, August, 1998

15. C. Deeney, T.J. Nash, R.B. Spielman, J.F. Seaman, J.S. McGurn, D.O. Jobe, M.F. Vargas, T.L. Gilliland, R.C. Mock, K.W. Struve, K.G. Whitney, P.E. Pulsifer, J.P. Apruzese, J.W. Thornhill, and J. Davis, Phys. Plasmas, 5, No. 6, June, 1998, p 2431

16. R. B. Spielman, C. Deeney, G. A. Chandler, M. R. Douglas, D. L. Fehl, M. K. Matzen, D. H. MeDaniel, T. J. Nash, J. L. Porter, T. W. L. Sanford, J. F. Seamen, W. A. Stygar, K. W. Struve, S. P. Breze, J. S. McGurn, J. A. Torres, D. M. Zagar, T. L. Gilliland, D. O. Jobe, J. L. MeKenney, R. C. Mock, M. Vargas, T. Wagoner, and D. L. Peterson, Bull. Amer. Phys. Soc., 41, No. 7, November, 1997, p. 1422

17. R.B. Spielman, C. Deeney, G.A. Chandler, M.R. Douglas, D.L. Fehl, M.K. Matzen, D.H. McDaniel, T.J. Nash, J.L. Porter, T.W.L.Sanford, J.F. Seaman, W.A. Stygar, K.W. Struve, S.P. Breeze, J. S. McGurn, J.A. Torres, D.M.Zagar, TI.L. Gilliland, D.O.Jobe, J.L. Mckenney, R.C. Mock, M.Vargas, and T.Wagoner, PBFA Z: A 60-TW//5-MJ Z-Pinch Driver, Proceedings of the 4 th International Conference on High-Density Z-pinches, Vancouver, Canada, May, 1997, American Institute of Physics, Woodbury, New York, 1997, p. 101 
18. D.L. Fehl, G.A. Chandler, F. Biggs, A.R. Moats, and R.J. Leeper, Rev. Sci. Ins., 68, No. 1, p 843,1997

19. D.L. Peterson, R.L. Bowers, J.H, Brownell, A.E. Greene, K.D. McLenithan. T.A. Oliphant, N.F. Roderick, and A.J. Scannapicco. Phys. Plas. 3, (1). 386, 1996

20. D.D. Ryutov, in preparation for Reviews of Modern Physics. "The Physics of Fast ZPinches" 


\section{List of Figures}

1. Schematic of the load hardware on $Z$ for $z p 214$ showing a nested wire array imploding onto an annular target. A gold half-moon fiducial is placed on the back wall of the hohlraum to verify that the inside of the hohlraum remains optically thin.

2. Radially resolved radiation temperatures parametric in time for $\mathrm{zp} 214$ unfolded from the axial time-resolved pinhole camera, $\mathrm{XRD}$ s and bolometer. At a diameter of $1.6 \mathrm{~mm}$ the tungsten inner wall has reached a radiation temperature of $180 \mathrm{eV}$.

\section{..}

3. Inner wall radiation temperature and diameter versus time for $z p 214$. At a diameter of $1.6 \mathrm{~mm}$ the tungsten inner wall has reached a radiation temperature of $180 \mathrm{eV}$.

4. Cavity radiation impingent on a $1.6 \mathrm{~mm}$ diameter pellet surface and cavity diameter versus time for $\mathrm{zp} 214$. At a pinch diameter of $1.6 \mathrm{~mm} 15 \mathrm{~kJ}$ of radiation would have struck the pellet surface.

5. Radiation temperature of the inside wall and outside wall of the dynamic hohlraum for zp214. At a temperature of 160 to $170 \mathrm{eV}$ the wall burns through and radiation burst out the side rises at $531 \mathrm{~ns}$. 
6. Power radiated out the axis measured and simulated for $z p 214$. The simulation gives sharper radiation bursts than the data. The difference is in part because the simulation does not include filamentation due to the use of a slotted current-return can.

7. Azimuthal line-out from a time-resolved pinhole camera for zp 113 taken as the implosion reaches a $4 \mathrm{~mm}$ diameter. Filaments are seen to occur roughly every 40 degrees in accordance with the nine-fold symmetry of the current return can.

8. Comparison of power radiated out the axis for a nested wire array imploding onto a gold foil with a slotted and with a solid current-return can. The improvement in power for this configuration with a solid can is a factor of 1.7. 9. Schematic of the load hardware on $Z$ for $z p 297$ showing a nested wire array imploding onto a cylindrical foam target. The foam has a density of $14 \mathrm{mg} / \mathrm{cc}$ and weighs $3 \mathrm{mg}$.

10. Radially resolved radiation temperatures parametric in time for $\mathrm{zp} 297$ unfolded from the axial time-resolved pinhole camera, XRDs and bolometer. The radiation temperature has reached $230 \mathrm{eV}$ at a diameter of $800 \mu \mathrm{m}$.

11. Comparison of time-integrated crystal spectra for zp214 and zp297. Zp297 shows a much hotter electron temperature of $2 \mathrm{keV}$ as compared to $0.7 \mathrm{keV}$ for $z p 214$. The difference is likely due to the enhanced stability of $z p 297$ due to a 
solid current-return can, allowing the pinch to reach higher density, which allows the electrons and ions to equilibrate.

12. Dynamic hohlraum diameter and temperature versus time for $\mathrm{zp} 214$ and zp297. Both hohlraums implode at $35 \mathrm{~cm} / \mu \mathrm{s}$. The temperature of $\mathrm{zp} 214$ is up earlier with a slower rate of rise. The temperature of $\mathrm{zp} 297$ rises very rapidly near the end of the compression.

13. The scaling of dynamic hohlraum radiation temperature with machine current. Data points are at 3.5 MA (ANGARA), 7 MA (SATURN), and 20 MA (Z). Temperatures are taken at a dynamic hohlraum diameter of approximately $1 \mathrm{~mm}$. The fitted curve is very close to a square root - dependence. 


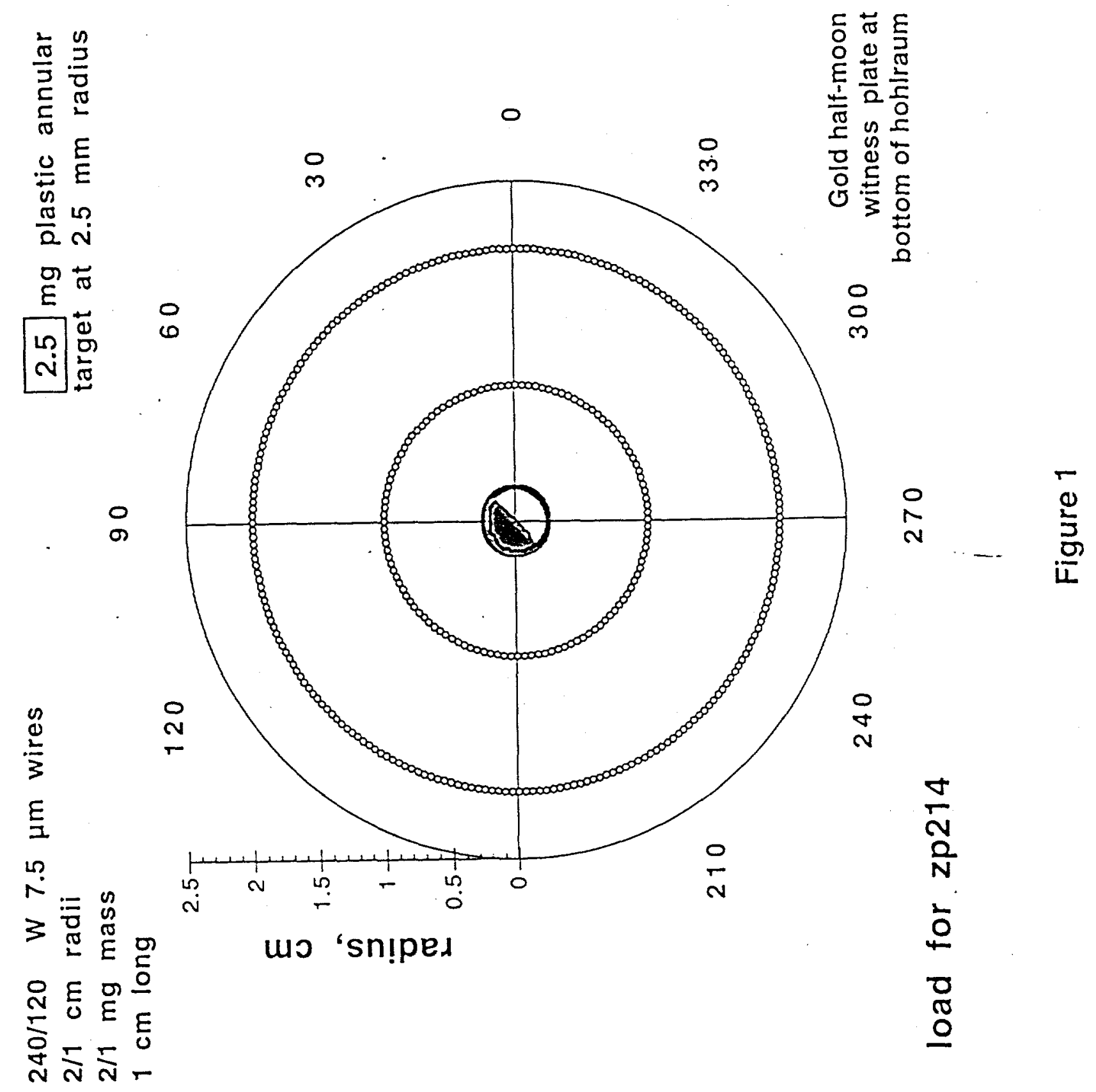




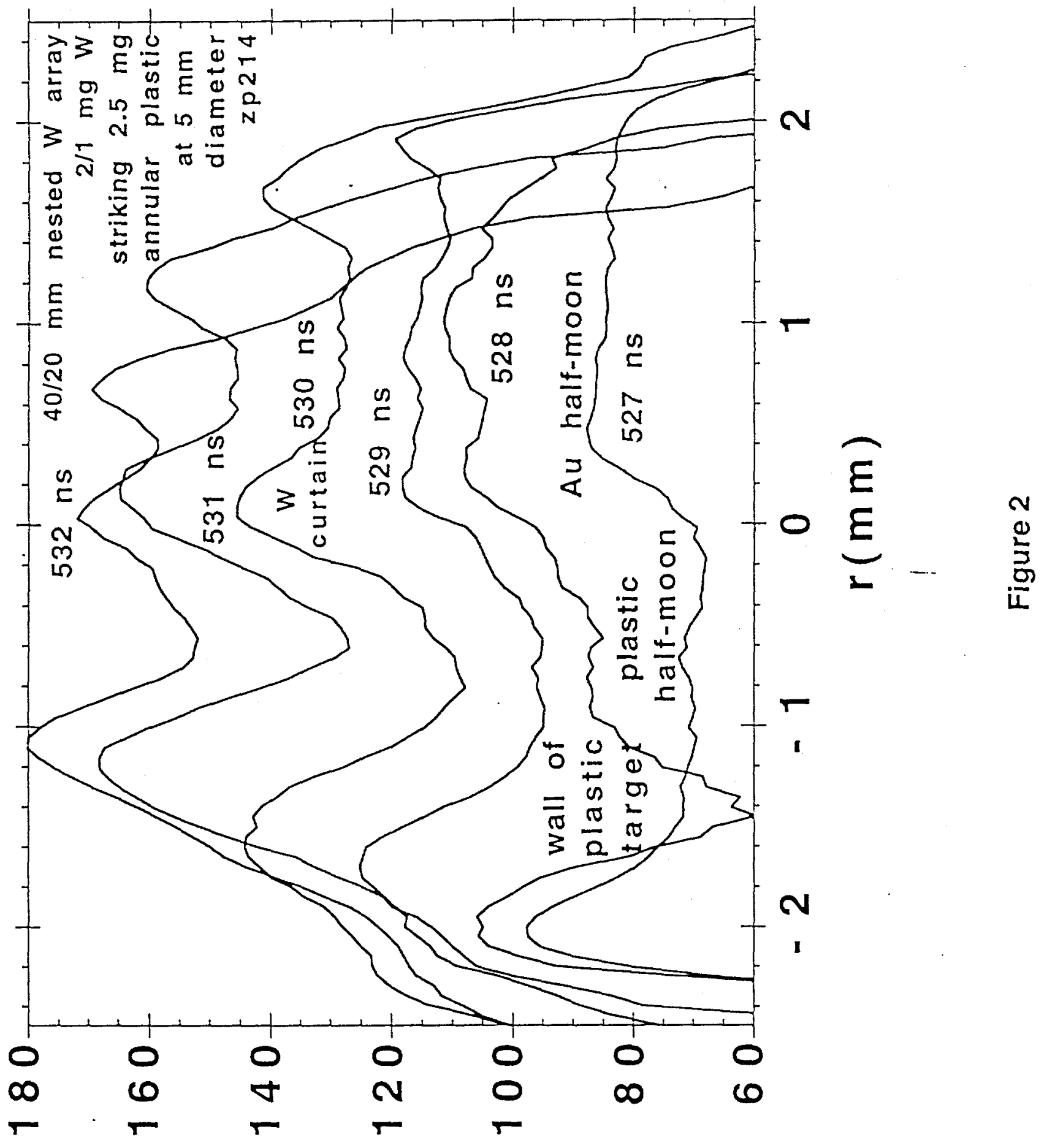




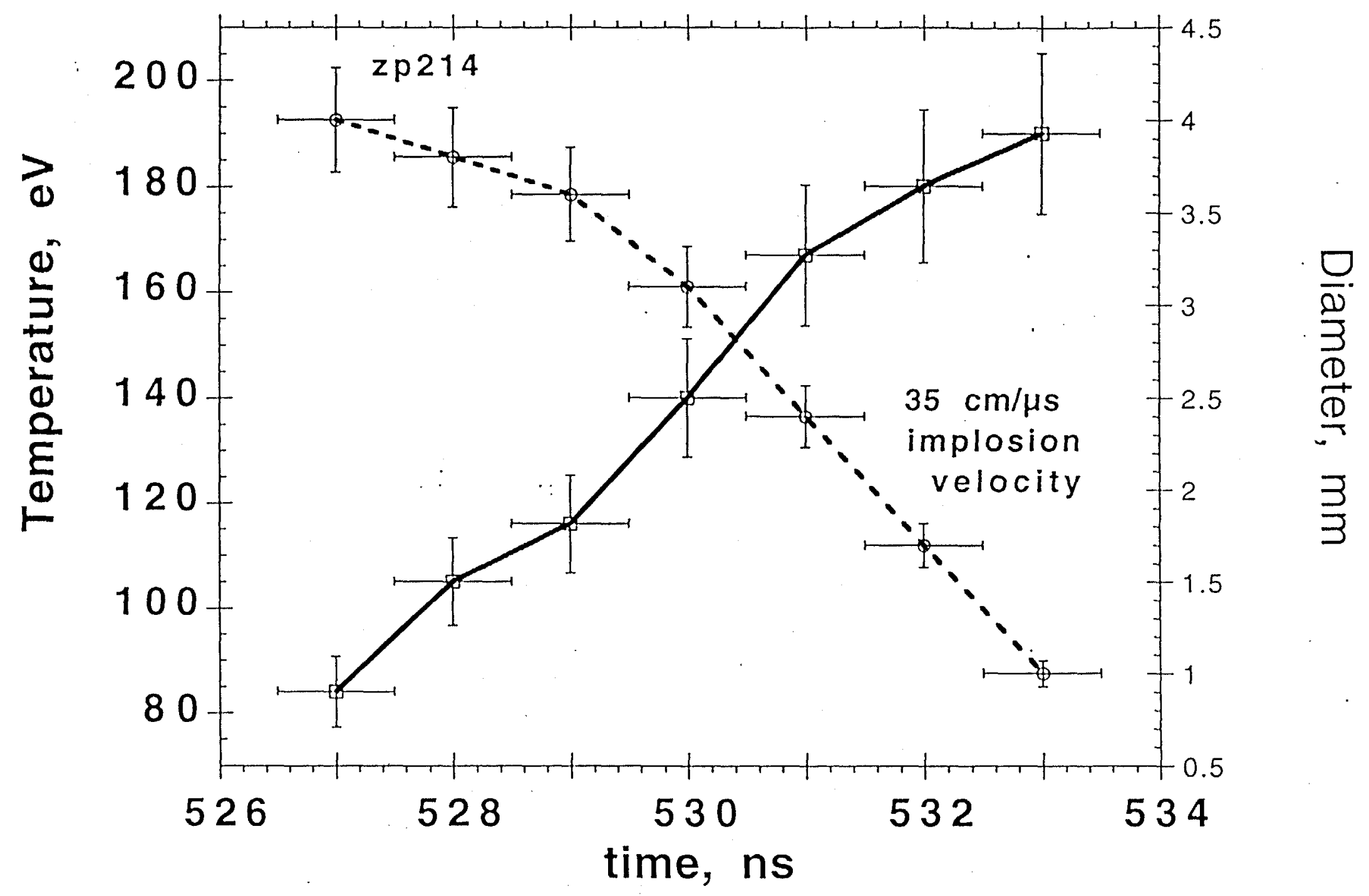

Figure 3 


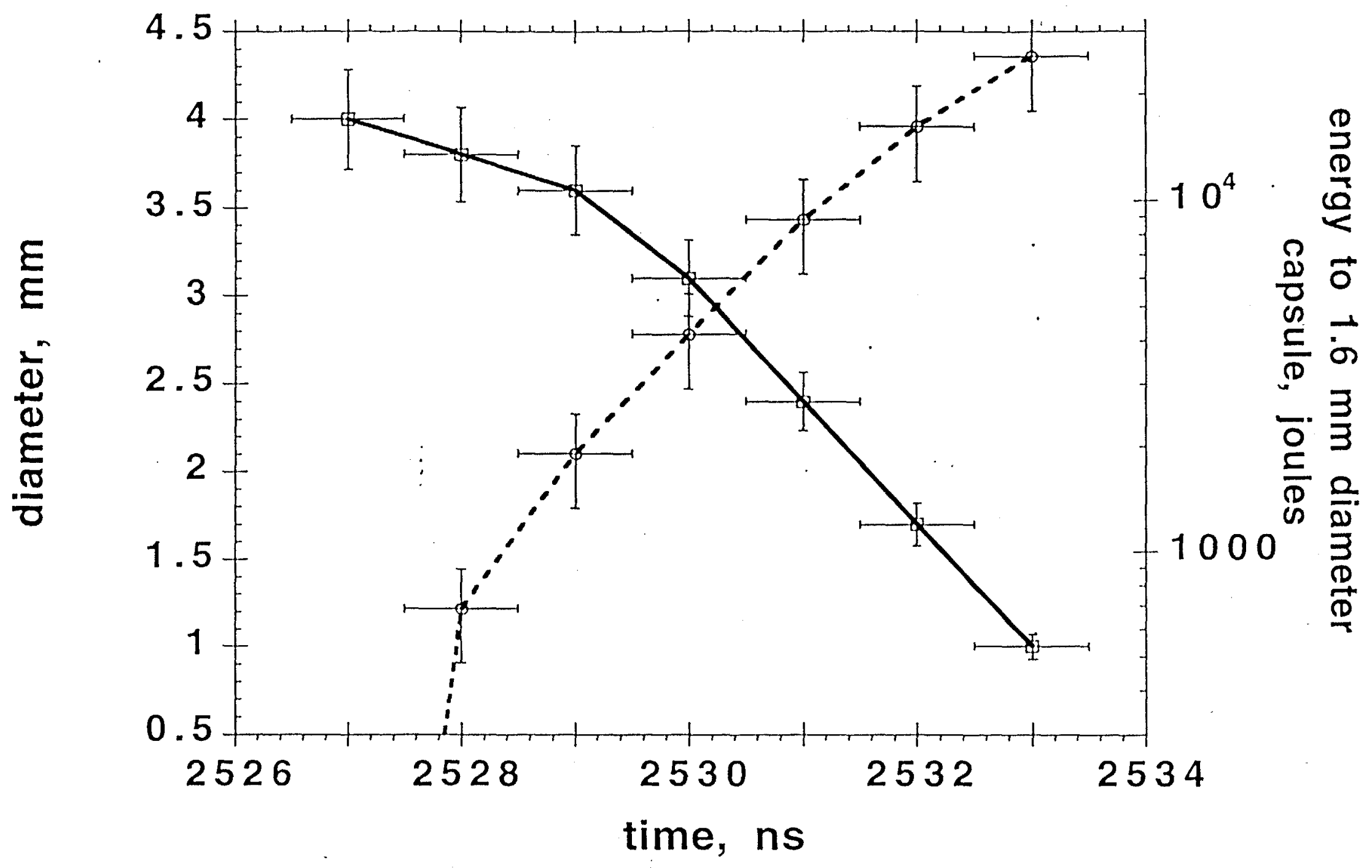

Figure 4 


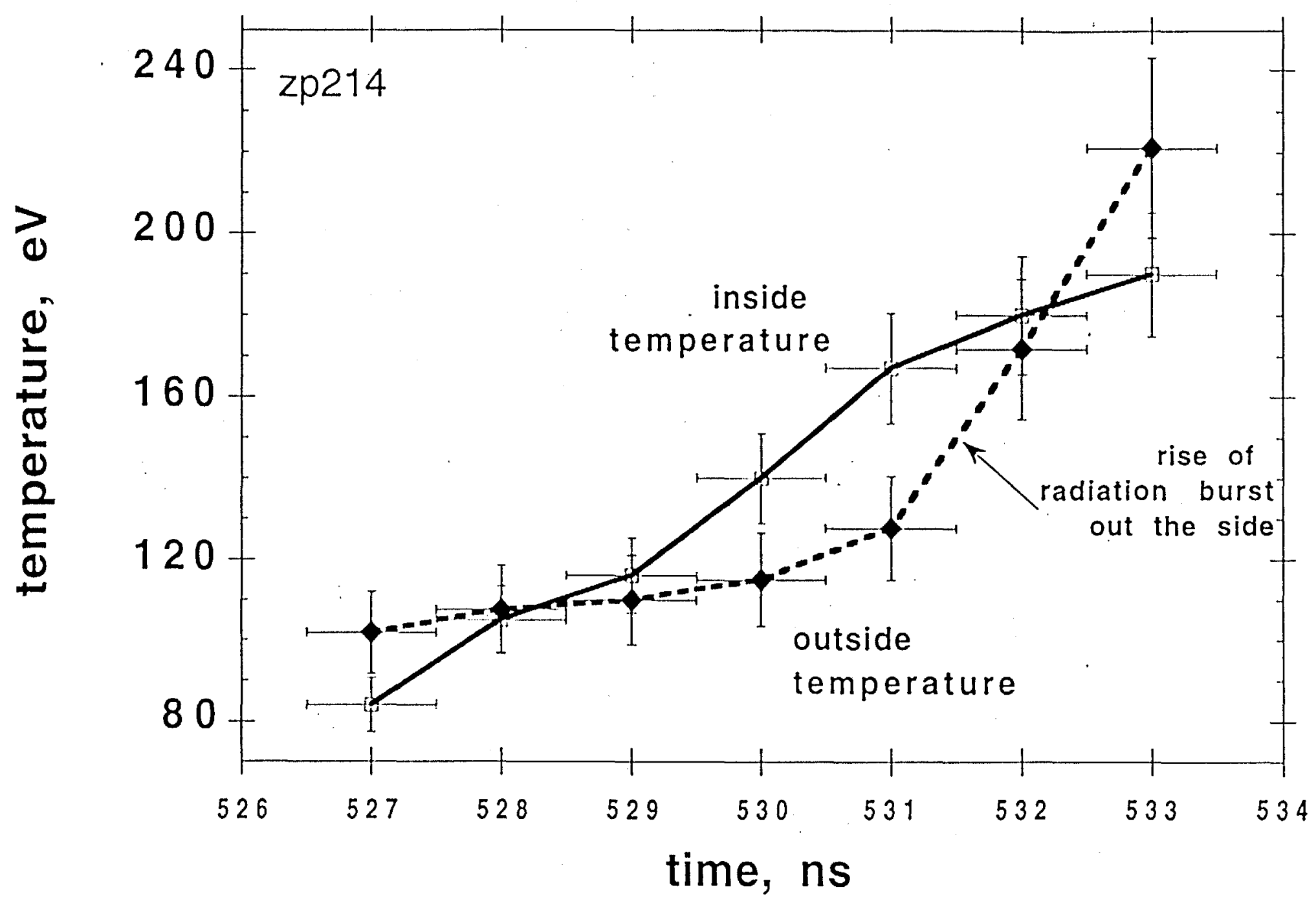

Figure 5 


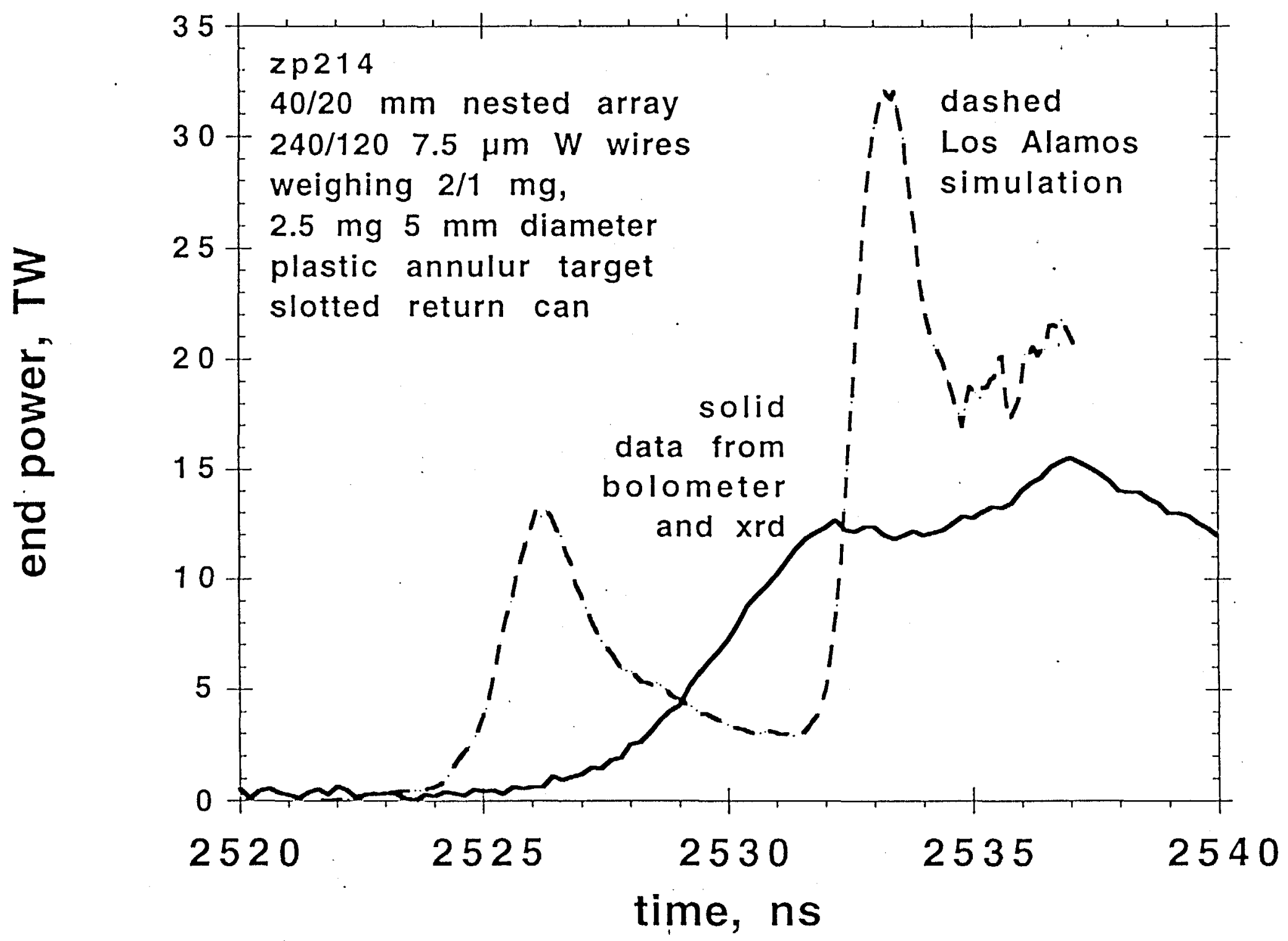

Figure 6 


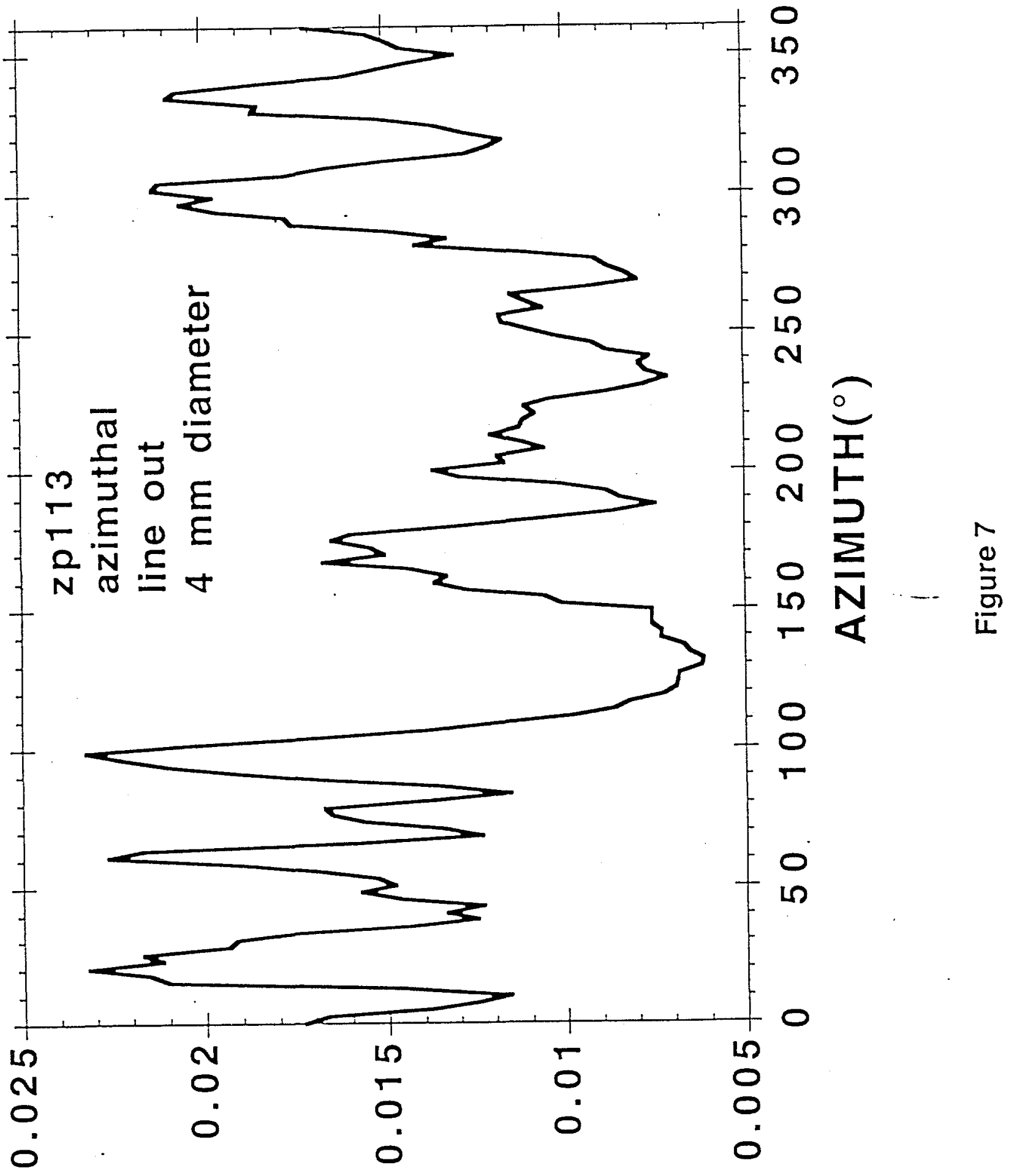

そயग/sб. 


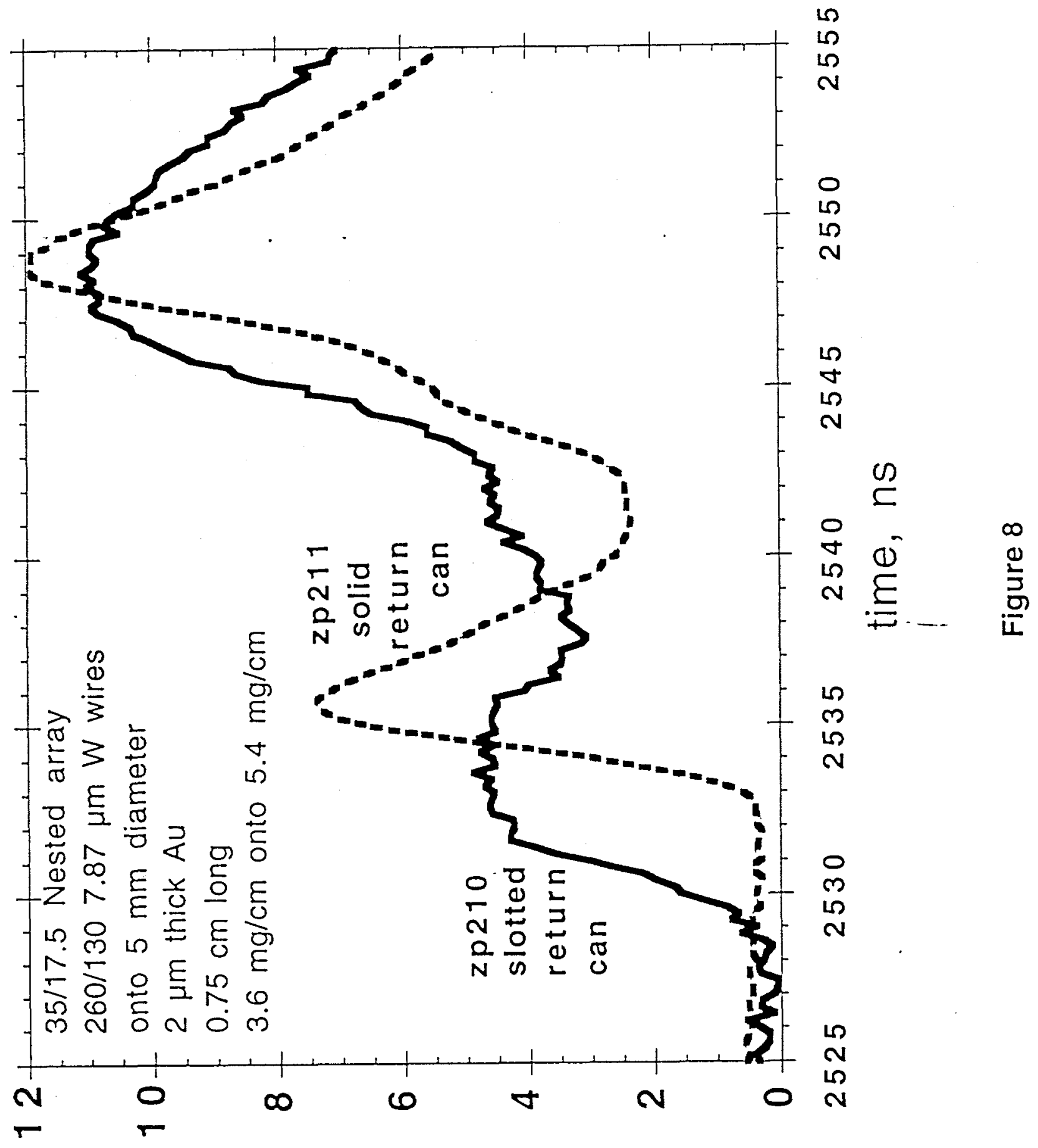

ML "ләмод рuә 
240/120 W $7.5 \mu \mathrm{m}$ wires

$2 / 1 \mathrm{~cm}$ radii

$2 / 1 \mathrm{mg}$ mass

$1 \mathrm{~cm}$ long

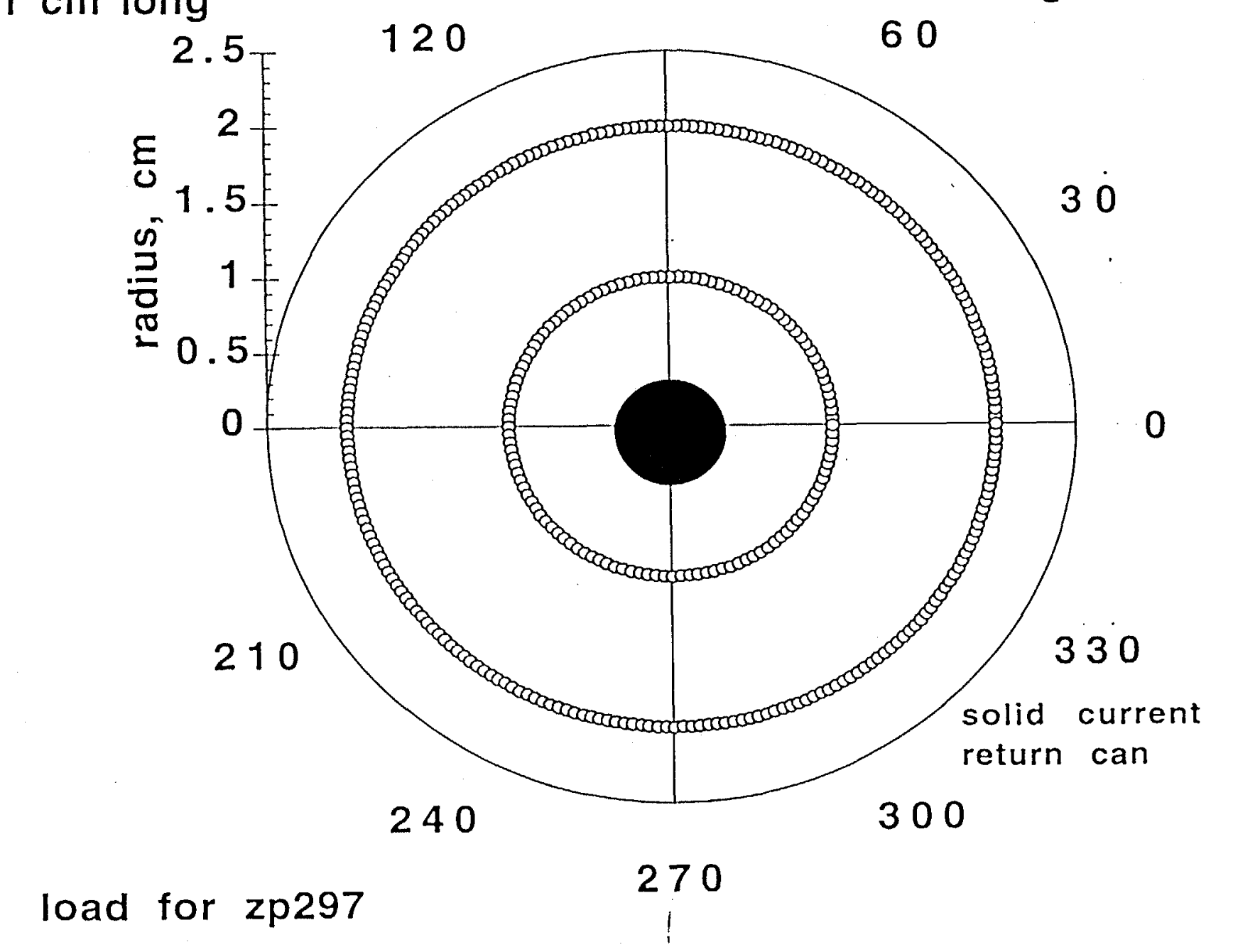

$3 \mathrm{mg}$ cylindrical foam target at $2.5 \mathrm{~mm}$ radius

$90 \quad 14 \mathrm{mg} / \mathrm{cc}$ foam

Figure 9 


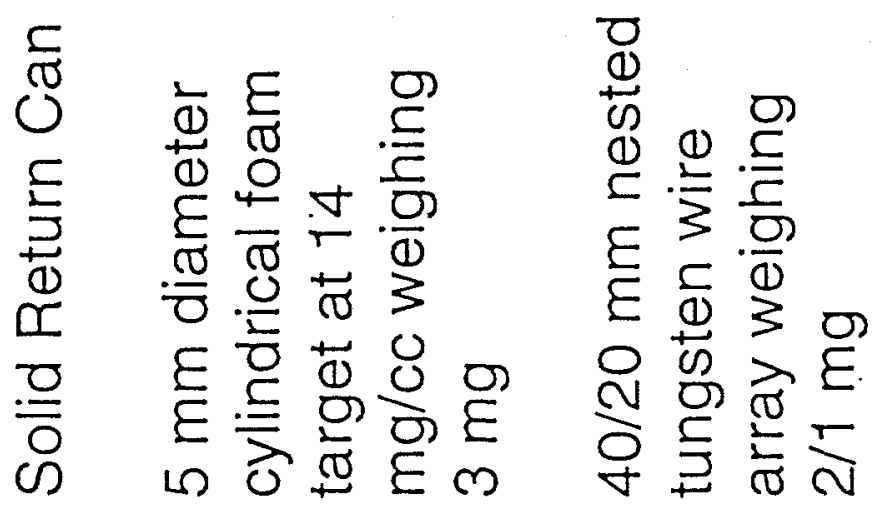

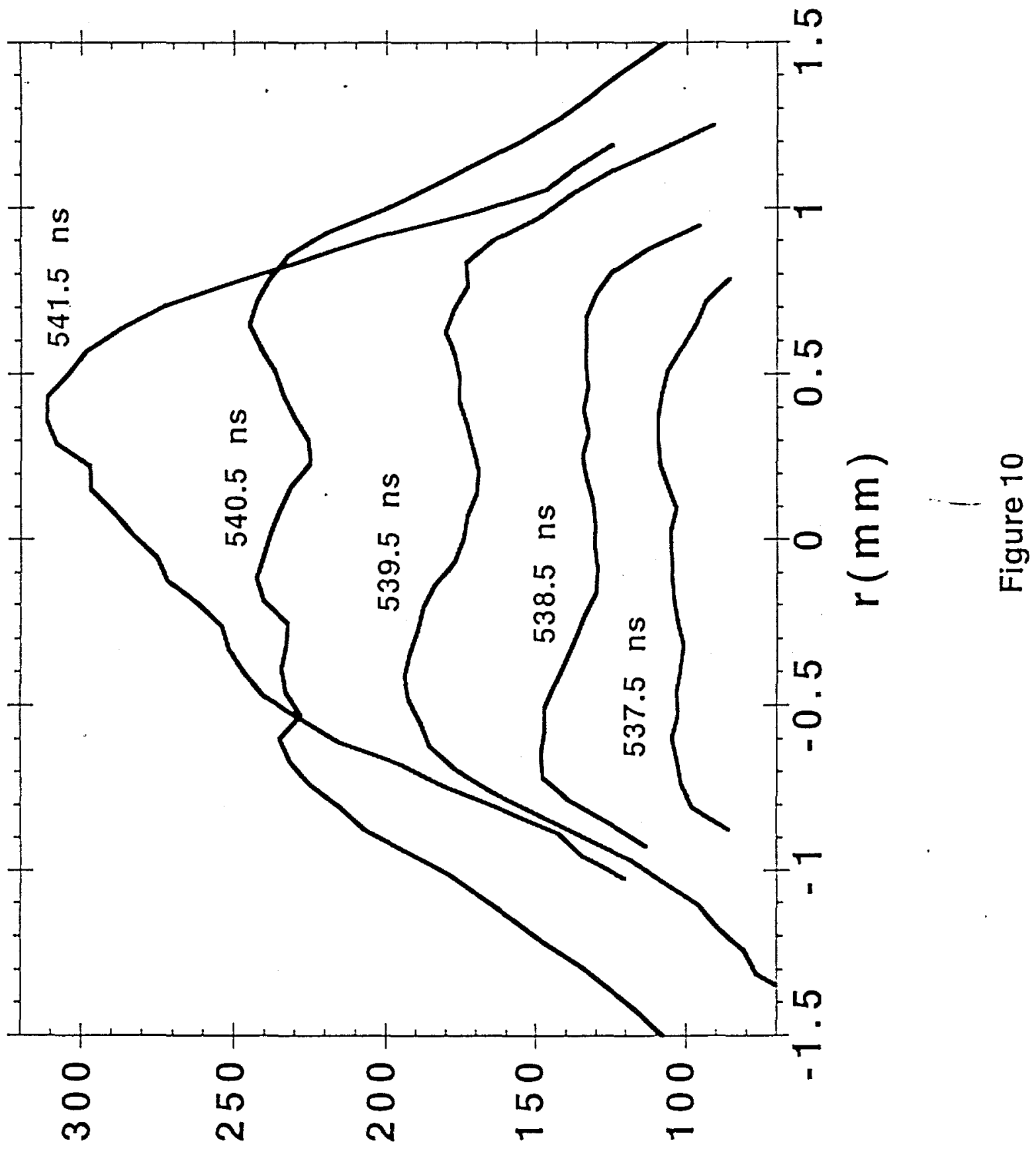




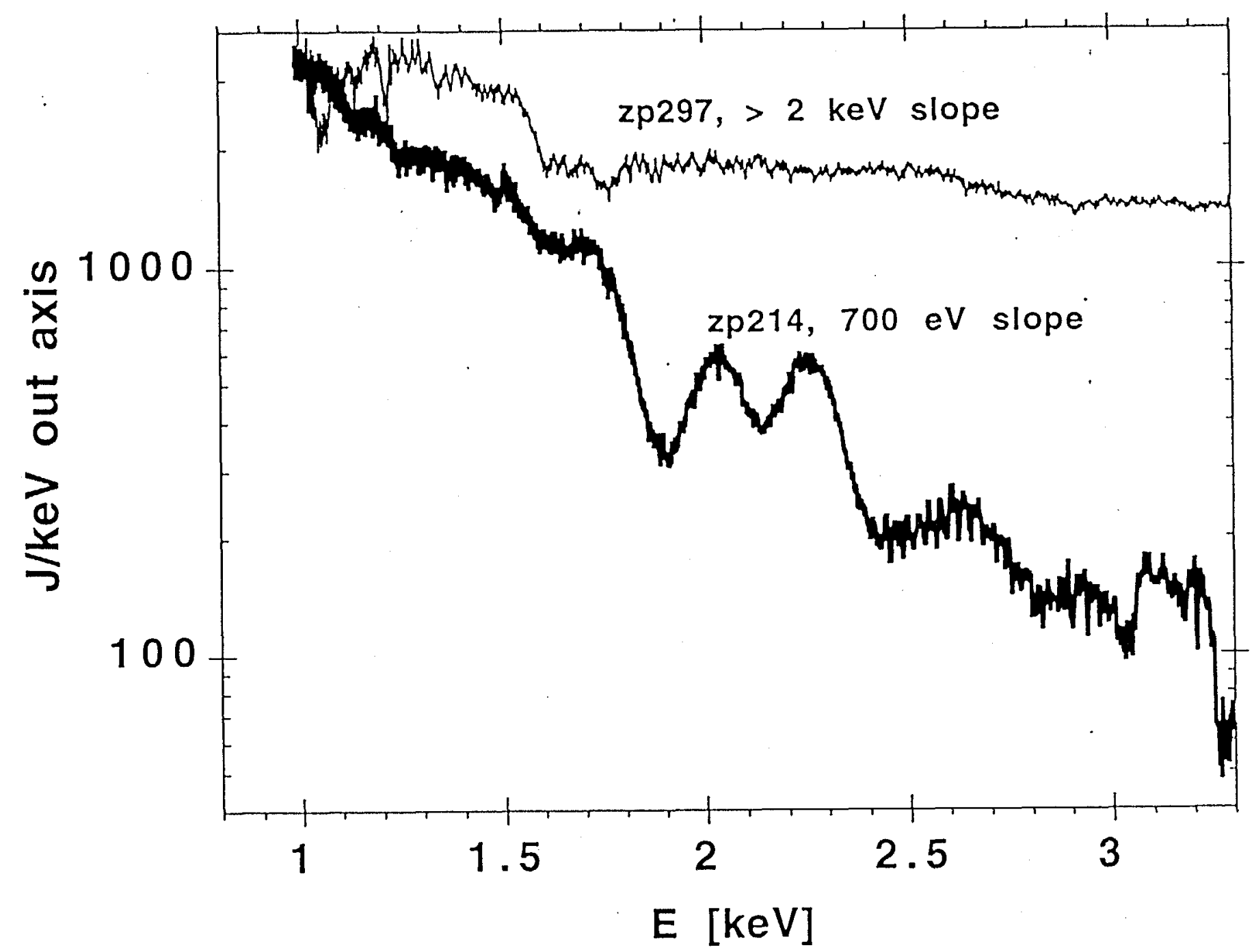

Figure 11 


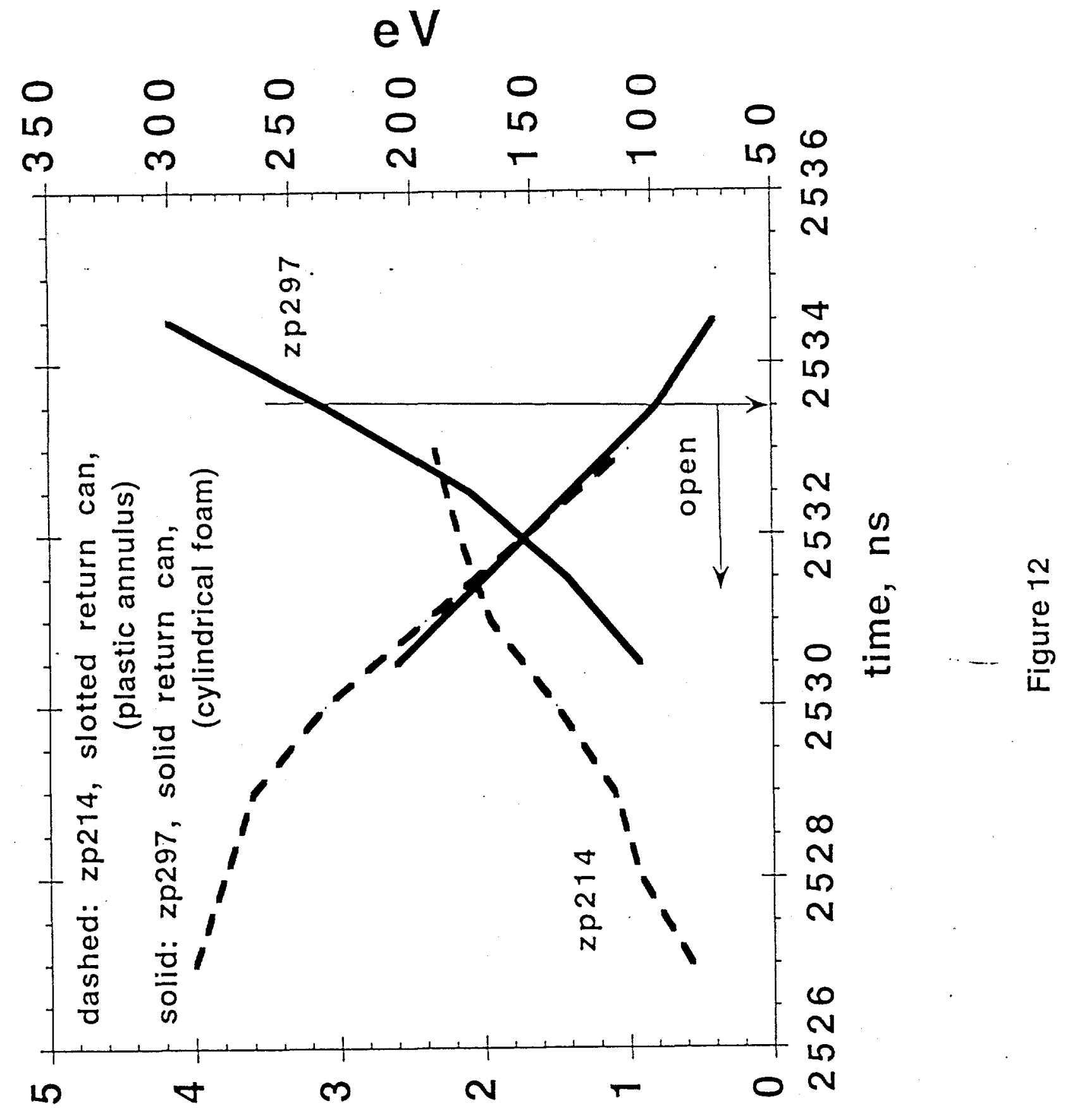

щu '^әңәше!p 


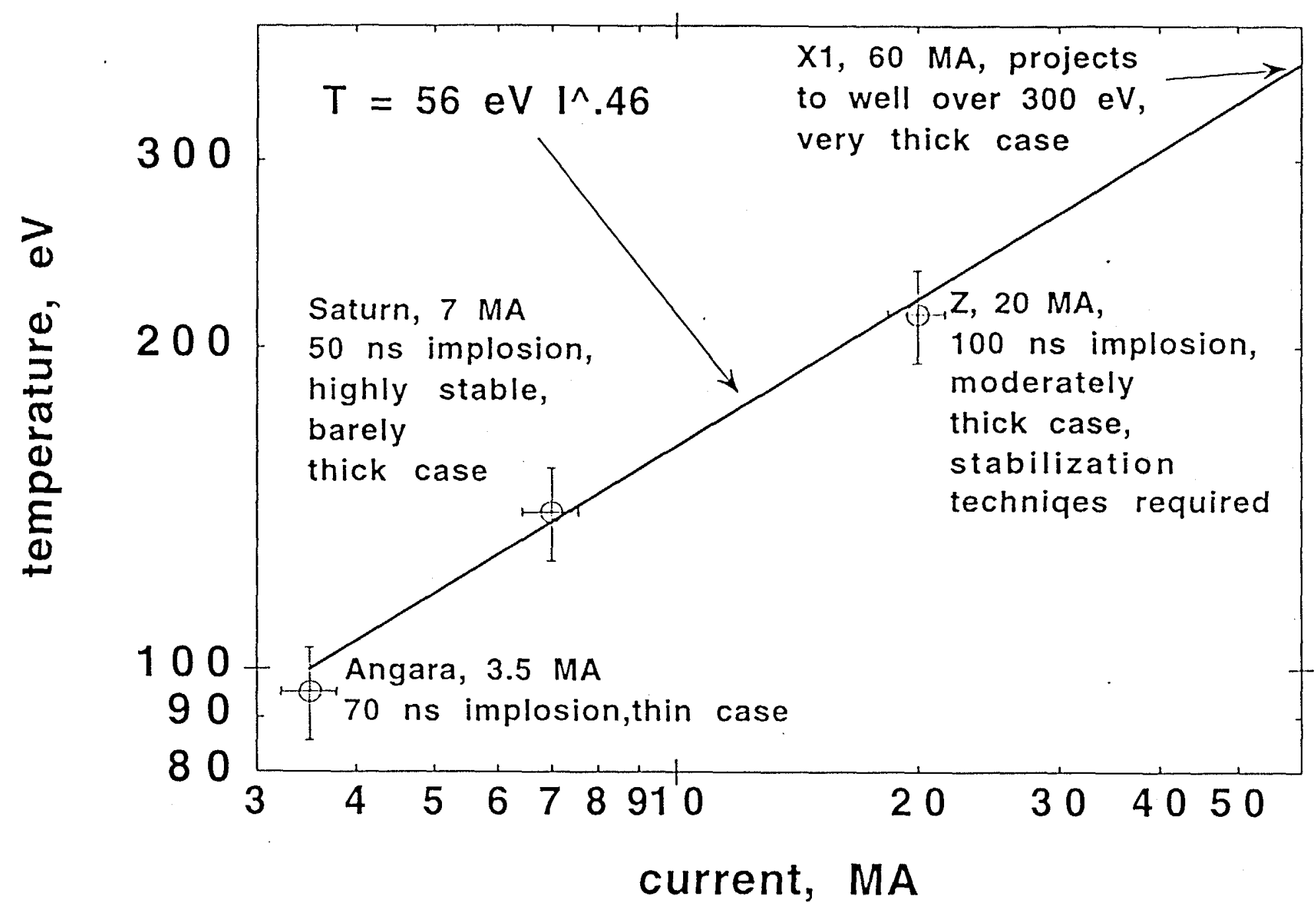

Figure 13 